\title{
GMR
}

\section{Bone morphogenetic protein 9 facilitates osteocarcinoma cell apoptosis and inhibits in vivo tumor growth}

\author{
J. Zhang ${ }^{1}$, J.H. Liang ${ }^{2}$ and J.G. Huang ${ }^{1}$ \\ ${ }^{1}$ Department of Oncology, Urumqi General Hospital of Lanzhou Military \\ Area Command of Chinese PLA, Urumqi, China \\ ${ }^{2}$ Department of Orthopedics, Urumqi General Hospital of Lanzhou Military \\ Area Command of Chinese PLA, Urumqi, China \\ Corresponding author: J.G. Huang \\ E-mail:dcthuang@163.com
}

Genet. Mol. Res. 15 (3): gmr.15038036

Received December 17, 2015

Accepted January 15, 2016

Published September 16, 2016

DOI http://dx.doi.org/10.4238/gmr.15038036

Copyright (C) 2016 The Authors. This is an open-access article distributed under the terms of the Creative Commons Attribution ShareAlike (CC BY-SA) 4.0 License.

\begin{abstract}
Osteosarcoma (OS) causes millions of death worldwide and, since there is no effective therapy, it is necessary to identify the molecular mechanism of OS, which can direct the development of new therapies. This study investigated the role of bone morphogenetic protein 9 (BMP9), a member of the transforming growth factor (TGF)- $\beta$ family, in OS development. This study first examined BMP9 expression in tissue from OS patients and normal subjects. The OS cell line (MG63) and tumor cells from OS patients were then transfected with BMP9 and cell proliferation and apoptosis were assessed. Western blot and reverse transcription-polymerase chain reaction were used to study the expression of cancer-related genes [B cell lymphoma (Bcl)-2, cleaved Caspase-3, Caspase-9, and poly ADP-ribose polymerase]. To confirm the in vivo impact of BMP9, mice were transplanted with OS tumor cells
\end{abstract}


and then treated with BMP9 carried in attenuated Salmonella enterica serovar Typhimurium. Our study found that the OS tumor tissue had a lower expression of BMP9 compared to normal tissue. Transfection of BMP9 in OS and MG63 cells inhibited cell growth and promoted apoptosis. In vitro studies showed a decrease in Bcl-2 gene expression and an increase in Cyto-c, Caspase-3, and Caspase-9 expression. In vivo studies indicated that consistent treatment with BMP9 in OS mice results in inhibition of tumor growth. This study shows that BMP9 inhibition is associated with OS development and that enhanced expression of BMP9 may be a potential treatment method for OS.

Key words: Bone morphogenetic protein 9; Osteocarcinoma cell; Transfection; Tumor

\section{INTRODUCTION}

Osteosarcoma (OS) is a very lethal type of tumor that causes millions of death all over the world. OS is divided into three groups depending on the histological characteristics: osteoblastic, chondroblastic and fibroblastic. Current treatment of OS mainly relies on the use of different chemotherapies (Bielack et al., 2009). A low survival rate of OS patients exists due to the limited efficiency of these therapeutic agents (Saeter et al., 2005); therefore, it is necessary to explore new strategies for treating OS.

Bone morphogenetic proteins (BMPs) are a group of proteins that belong to the transforming growth factor- $\beta$ (TGF- $\beta$ ) superfamily (Carreira et al., 2014). Current studies have found that BMPs (e.g., BMP2, BMP6, BMP7, BMP9) are associated with osteogenic differentiation (Higuchi and Yoshikawa, 2004). In particular, BMP9 was reported to be involved in a series of cellular processes, including morphogenesis, hematopoiesis, glucose homeostasis, and neuronal differentiation, by forming heterotetrameric receptor complexes (Chen et al., 2003). These complexes can phosphorylate the activin receptor-like kinase (ALK1-7) and activate downstream signaling restricted Smad proteins (e.g., R-Smads, Smads1, 5, and 8), which are then translocated to the nucleus and function as regulators of gene transcription. In particular, BMP9, one of the most potent members of the BMP family, was found to play a vital role in cancer development. For example, one study found that BMP9 can inhibit the proliferation and invasion of prostate cancer (Wang et al., 2011). A similar study also reported on the association between prostate and ovarian cancer migration and BMP9 (Ye et al., 2008; Herrera et al., 2009). Although one study reported the association between BMP9 and proliferation of OS cell lines ( $\mathrm{Lv}$ et al., 2013), little is known about the role of BMP9 in OS tumor tissues from clinical patients, particularly with respect to its interaction with oncogenes. It is also necessary to investigate the role BMP9 in animal models of OS.

In this study, the expression of BMP9 in OS tissues from patients and tissue from healthy subjects was studied. Our study found decreased expression of BMP9 in OS tissues compared to that from healthy subjects. To investigate the role of BMP9 in OS disease, a human OS cell line and cells from OS patient tumor tissues were transfected with BMP9 and proliferation, apoptosis and cell cycle were assessed. In addition, western blot was used to examine the association between BMP9 and oncogene (Cyto-c, Caspase 9, and Caspase3) expression in all three types of cells. To assess the in vivo anti-cancer effect of BMP9,

Genetics and Molecular Research 15 (3): gmr.15038036 
C57BL/6J mice were transplanted with MG63 tumor cells, treated with BMP9 carried in Salmonella enterica serovar Typhimurium, and then tumor growth was monitored.

\section{MATERIAL AND METHODS}

\section{Tissue and cells}

Studies that involve animals and human tissues are under the regulation of the Institutional Review Board of Urumqi General Hospital of Lanzhou Military Area Command of Chinese PLA. Patient tissues were collected from patients who have been diagnosed with OS. The patients were notified and signed written consent forms for the use of the tumor tissues in the current study. The tissue samples were stored in liquid nitrogen and protected with paraffin film until use. MG62 cells were obtained from American Tissue Type Collection (Manassas, VA, USA). RPMI 1640 enriched with 10\% fetal bovine serum, penicillin (100 U/ $\mathrm{mL})$ and streptomycin $(100 \mathrm{mg} / \mathrm{mL})$ was used for cell culture (VWR, USA).

\section{Gene transfection}

To investigate the role of BMP9 in cell function, MG63 cells and homogenized cells from patient tissue were transected either with pcDNA3.1 or pcDNA3.1-BMP9 plasmids (Shanghai Blue Gene Biotech, China) and Lipofectamine 2000 (Roche, Switzerland). The transfection procedures were performed by following the manufacturer instructions. In brief, a given amount (e.g., $2 \mu \mathrm{g}$ ) of pcDNA (pcDNA3.1 or pcDNA3.1-BMP9) was mixed with $9 \mu \mathrm{L}$ cell culture medium and $5 \mu \mathrm{L}$ Lipofectamine 2000. The mixture was added into cells cultured in cell culture plates $\left(1 \times 10^{5}\right.$ cells per well) followed by culturing at $37^{\circ} \mathrm{C}$ and $5 \% \mathrm{CO}_{2}$ for 24 or $48 \mathrm{~h}$.

\section{Proliferation assay}

Cell proliferation was studied with the MTT colorimetric assay by following the manufacturer instructions. In brief, cells transfected with plasmid DNA were cultured on 96well plates at a cell density of $5 \times 10^{4}$ cells per well. MTT $(25 \mu \mathrm{L})$ in PBS was added into the cell culture plate and incubated under cell culture conditions for $4 \mathrm{~h}$, followed by the addition of $150 \mu \mathrm{L}$ dimethyl sulfoxide (Sigma, USA). The cells were placed on a shaker for $5 \mathrm{~min}$ before assessment with a microplate reader (Bio-Rad, Richmond, CA, USA) at $570 \mathrm{~nm}$.

\section{Reverse transcription-polymerase chain reaction (RT-PCR)}

RNA was extracted from cells using Trizol (Invitrogen, USA) and the All Total Nucleic Acid Isolation Kit was used (Ambion Inc., USA). The experiment was performed by following the manufacturer's instructions. The following components were employed for PCR analysis: $10 \mu \mathrm{g}$ total RNA, $10 \mathrm{mM}$ dNTP (3 mg), oligo dT (0.5 mg), RNasin (20 U), and M-MLV reverse transcriptase (200 U, Promega Corp., USA). The above components were made to a total volume of $10 \mu \mathrm{L}$ with water. The PCR was performed using the following protocol: $95^{\circ} \mathrm{C}$ for $3 \mathrm{~min} ; 25-30$ cycles of $95^{\circ} \mathrm{C}$ for $30 \mathrm{~s}, 55^{\circ} \mathrm{C}$ for $30 \mathrm{~s}$, and $72^{\circ} \mathrm{C}$ for $1 \mathrm{~min}$; and $72^{\circ} \mathrm{C}$ for $10 \mathrm{~min}$. The densitometry scan was performed with the Image Master VDS Software (Pharmacia Biotech, USA).

Genetics and Molecular Research 15 (3): gmr.15038036 


\section{Protein extraction and western blot}

Proteins were extracted from cells by following procedures reported in the literature (Dignam, 1990; Pang et al., 2009). The protein bands were separated by SDS-PAGE followed by transfer onto PVDF membrane (Millipore, Bedford, MA, USA). Antibodies used were rabbit anti-human B-cell lymphoma 2 (Bcl-2), Cyto-c, Caspase-3, cleaved Caspase-3, and $\beta$-actin (Abcam, USA) were employed for binding. The Quantity One software (Bio-Rad, Hercules, CA, USA) was used to analyze the intensity of the protein band.

\section{Flow cytometry}

For flow cytometry analysis, cells were collected and resuspended in PBS followed by staining with $5 \mu \mathrm{L}$ AnnexinV $(1 \mathrm{mg} / \mathrm{mL})$ (Beckman Coulter, Fullerton, CA, USA) at room temperature for $15 \mathrm{~min}$. The cells were then stained with propidium iodide $(1 \mathrm{mg} / \mathrm{mL})$ for 5 min before running the flow cytometry assay.

\section{In vivo anti-tumor study}

BALB/c nude (6-8 weeks old) mice were obtained from the Institute of Zoology, Chinese Academy of Sciences, Beijing, China. To establish tumor burden on mice, 3 x $10^{5}$ cells were implanted in the right flank of mice. Tumor was observed after 7 days of cell implantation. The mice were then treated with different doses of pcDNA3.1-BMP9 or pcDNA3.1 (control) carried by the attenuated $S$. enterica serovar Typhimurium. The transfection was performed with the electronic method as reported in the literature (Lerman and Minna, 2000). In brief, plasmid pcDNA3.1 or pcDNA3.1-BMP9 at a density of $10^{8}$ colony-forming units per $45 \mu \mathrm{L}$ were injected into mice through tail vein. Mice were injected every day after the establishment of the tumor. Tumor size was measured daily and mice were sacrificed when the tumor size reached 150-200 $\mathrm{mm}^{3}$. All studies that involve animals followed the regulation rules of Animal Care Committee in Urumqi General Hospital of Lanzhou Military Area Command of Chinese PLA.

\section{RESULTS}

\section{BMP9 expression suppression in OS cancer tissues}

We started our study by investigating the levels of BMP9 mRNA in OS cancer tissues, cells from healthy subjects, and MG63 cells. Figure 1A shows the representative mRNA levels of BMP9 in the three types of cells as analyzed by RT-PCR. Compared to normal tissues, there is a slight reduction in BMP9 in cells from cancer tissues and MG63 cells (Figure 1A, middle bar). We quantified the expression of BMP9 in the three different cell types as well and, when compared to normal tissue, OS cells have a $40 \%$ reduction and MG63 cells have a 70\% reduction in BMP9 (Figure 1B). To quantify the expression of BMP9 proteins, western blots were employed. Similar to RT-PCR analysis, the OS and MG63 cells have a reduction in the expression of BMP9 proteins (Figure 1C). Quantification of the BMP9 protein reduction in OS and MG63 cells, as compared to normal tissues, are similar to the trend observed in RT-PCR (Figure 1D).

Genetics and Molecular Research 15 (3): gmr.15038036 
A

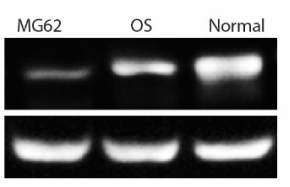

C

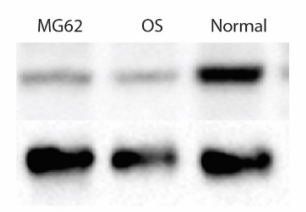

$B$
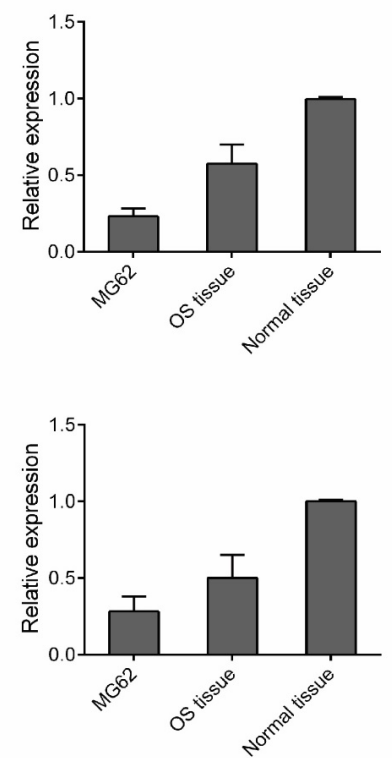

Figure 1. Expression of BMP9 in MG62 cells, OS cells, and normal tissue cells. A. RT-PCR analysis and B. relative expression of BMP9. C. Western blot analysis and D. relative expression of BMP9 expression. The expression of BMP9 in normal tissue was used as a standard.

\section{BMP9 inhibits OS cancer cell growth and promotes apoptosis}

To study the in vitro function of BMP9, pcDNA3.1-BMP9 or pcDNA3.1 was transfected into OS and MG63 cells (Figure 2A). Cell proliferation and apoptosis were monitored $48 \mathrm{~h}$ after transfection. To confirm the successful expression of BMP9 in these cells, RT-PCR was employed. Figure 2A shows the RT-PCR analysis of cells transfected with pcDNA3.1 or pcDNA3.1-BMP9. The presence of the BMP9 band indicates the transfection of pcDNA3.1-BMP9 cDNA into OS cells. We next analyzed growth of cells by counting cell number over time. Our study found that OS cells transfected with pcDNA3.1-BMP9 are inhibited in their growth, whereas the cells transfected with pcDNA3.1 had normal growth over time (Figure 2B). Similar trends were also observed in MG62 cells, where the cells transfected with pcDNA3.1-BMP9 were inhibited in growth as compared to the ones transfected with pcDNA3.1. (Figure 2B) We also investigated apoptosis in the two types of cells. The MG62 cells transfected with pcDNA3.1-BMP9 had a increase in their apoptosis rate as compared to cells transfected with pcDNA3.1. The OS cells transfected with pcDNA3.1-BMP9 had a slight increase in their apoptosis (from 12\% to 19\%) as compared to OS cells transfected with pcDNA3.1 (Figure 2C).

\section{BMP9 results in enhanced expression of apoptotic proteins}

We next assessed the expression of tumor suppressor genes over time in OS or MG62 cells transfected with pcDNA3.1-BMP9 compared to OS cells transfected with pcDNA3.1 as the control. We first analyzed the expression of Bcl-2, which belongs to the Bcl-2 family and 
affects tumor development by regulating cell apoptosis. Compared to OS cells transfected with pcDNA3.1, there was a gradual increase in Bcl-2 expression over time in cells transfected with pcDNA3.1-BMP9 (Figure 3A and 3B).We next employed Western blot to analyze the expression of cytoplasmic cytochrome $\mathrm{c}$ (Cyto-c). Cyto-c is associated with mitochondrial apoptosis channels on the mitochondrial membrane and its expression is induced during morphological changes that occur during cell apoptosis (Dejean et al., 2006). Our study found that cells transfected with pcDNA3.1-BMP9 had a slight increase in the expression of Cyto-c 24 to $96 \mathrm{~h}$ after transfection (Figure 3A, second row). Caspase-9 is another downstream apoptotic gene that is activated in the mitochondria, following the activation of Caspase-3. There was an increase in the expression of these genes in OS cells transfected with pcDNA3.1BMP9 compared to cells transfected with pcDNA3.1 only (Figure $3 \mathrm{~A}$ and $\mathrm{B}$, third and fourth rows), indicating BMP9 affects apoptosis of cancer cells.
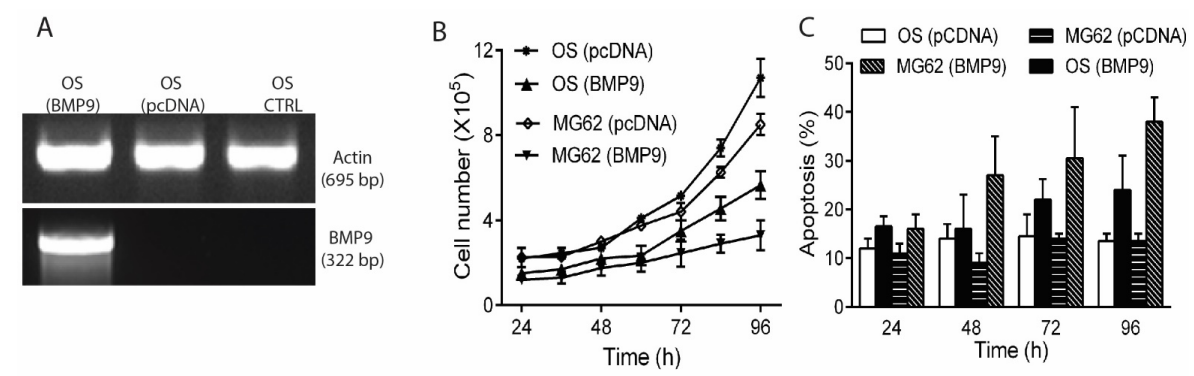

Figure 2. A. Confirmation of BMP9 transfection in OS cells treated with pcDNA3.1-BMP9. OS cells treated with pcDNA3.1 and cells without any treatment (CTRL) are used as controls. B. Effect of pcDNA3.1 and pcDNA3.1BMP9 (BMP9) on cell growth, as determined by number of cells counted over time. C. Apoptosis of OS and MG62 cells over time. The cells were transfected with pcDNA3.1-BMP9 (BMP9) or pcDNA3.1.

A

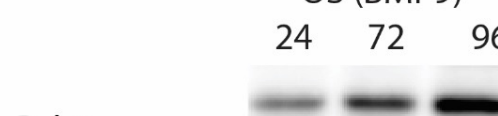

$\mathrm{BCl}-2$

Cyto-c

Caspase-9

Caspase-3

Actin
B

$\mathrm{BCl}-2$

Cyto-c

Caspase-9

Caspase-3

Actin

\section{OS (pcDNA)}
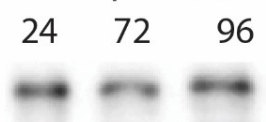

$\cdots$

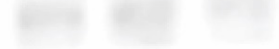

$--$
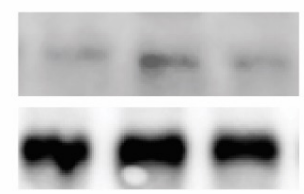

Figure 3. Expression of apoptosis-related proteins (Bcl-2, Cyto-c, Caspase-9 and Caspase-3) in OS cells transfected with pcDNA3.1-BMP9 (BMP9) (A) or pcDNA3.1 (B). The expression of $\beta$-actin (Actin) in both types of cells was used as a control. 


\section{BMP9 can inhibit tumor growth in mice}

We next assessed the effect of BMP9 in vivo by examining OS tumors in mice after treatment with Salmonella carrying pcDNA3.1 or pcDNA3.1-BMP9 (Mei et al., 2002). Our study found that while tumors keep growing in mice with both type of treatments, the mice treated with Salmonella carrying pcDNA3.1-BMP9 had a slower rate of growth in tumors than those treated with Salmonella carrying pcDNA3.1 (Figure 4A). Assessment of tumor weight on day 26 shows that there is an obvious difference in the weight of the tumor in the two groups of mice, where the mice treated with pcDNA3.1 had an average weight of $0.98 \mathrm{~g}$ and the mice treated with pcDNA3.1-BMP9 had an average tumor weight of $0.53 \mathrm{~g}$ (Figure 4B). To investigate the molecular mechanism behind this phenomenon, we analyzed the production of BMP9 and tumor apoptosis-related proteins in the tumor tissue. Our study found an enhanced production of BMP9 in tumor cells from mice treated with pcDNA3.1-BMP9, indicating a portion of BMP9 was delivered to the tumor site successfully (Figure 4C). We also analyzed the expression of Bcl-2, Cyto-c, Caspase-9 and Caspase-3. Again, no dramatic difference was found in the expression of Bcl-2 between the two groups of mice (Figure 4C). As for the production of other anti-tumor genes, there were similar trends. There is increased expression of Cyto-c, Caspase-9 in mice treated with pcDNA3.1-BMP9 compared to mice treated with pcDNA3.1 (Figure 4C). We also observed an increase in the production of Caspase-3 between the two groups of mice (Figure 4C).
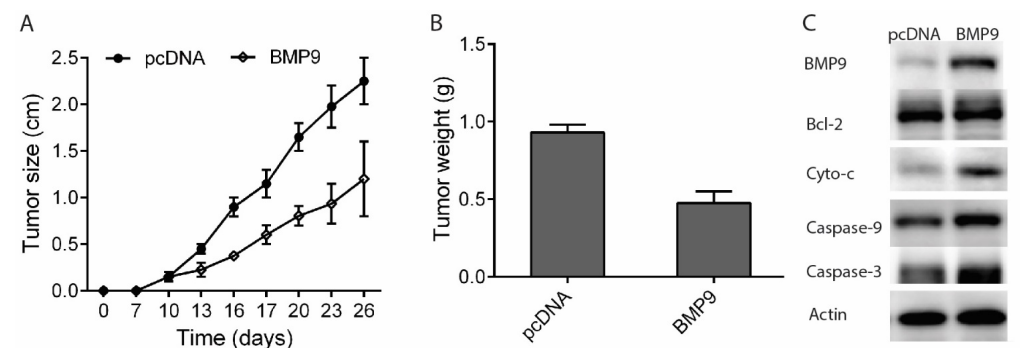

Figure 4. Impact of pcDNA-BMP9 (BMP9) transfection on tumor growth in mice. A. Tumor growth in mice treated with pcDNA3.1 or pcDNA3.1-BMP9. B. Weight of tumor in mice treated with pcDNA3.1 or pcDNA3.1BMP9. Tumor was dissected from mice on day 25 and weighed immediately after dissection. C. Expression of BMP9 and anti-cancer proteins in tumors from mice treated with pcDNA3.1 or pcDNA3.1-BMP9. The expression of $\beta$-actin (Actin) was used as a control.

\section{DISCUSSION}

OS is one of the primary malignant tumors that occur in children and young adults. This disease is aggressive, metastasizing mostly to the lungs and bones (Gill et al., 2013). It is therefore necessary to study and find strategies for treating this disease. As a member of TGF- $\beta$ superfamily, BMPs have been associated with cancer and many other diseases (Kim and Choe, 2011). BMP9 was first isolated from mouse liver and since then the potent role of BMP9 in inducing osteogenic differentiation has been examined (Song et al., 1995). In particular, recent studies found that BMP9 plays a role in tumor development. It was reported that BMP9 can inhibit the proliferation and invasiveness of breast cancer cells (Wang et al., 2011). Of note, many studies have reported the association between prostate and ovarian 
cancer migration and BMP9 expression (Ye et al., 2008; Herrera et al., 2009). Another study found that there is an association between BMP9 and proliferation in an OS cell line (Lv et al., 2013). Considering the importance of BMP9 and the necessity of developing new strategies for treating OS disease, we were interested in examining the use of BMP9 for treatment of OS.

We started our study by assessing the levels of BMP9 mRNA and proteins in OS patient tissues. We found that both were reduced compared to normal tissue (Figure 1). Studies by other groups have shown that BMP9 can inhibit the proliferation of the OS cell line and ovarian cancer cells (Lv et al., 2013). These results indicate that inhibition of BMP9 might be associated with the development of OS tumors. We next transfected two types of OS cell lines, MG63 cells and cells isolated from OS patient tissue, with pcDNA3.1-BMP9. Our study found enhanced expression of BMP9 in cells transfected with pcDNA3.1-BMP9, demonstrating the successful transfections of the plasmid DNA (Figure 2A). We also found that cells transfected with pcDNA3.1-BMP9 had decreased proliferation and apoptosis compared to cells transfected with pcDNA3.1 (Figure 2). Considering this, we assessed the expression of apoptosis-related genes in cells transfected with pcDNA3.1-BMP9. The apoptosis of cells involves intricate expression of a variety of oncogenes and tumor suppressor genes (Williams and Smith, 1993; Liebermann et al., 1995). Cyto-c is an oncoprotein released from the mitochondria of cells and it functions as an intermediate in apoptosis by inducing morphological changes in cells, which is usually followed by apoptosis (Liu et al., 1996; Dejean et al., 2006). Studies have found that the release of Cyto-c from the mitochondrial channel is increased when anti-cancer drugs are used to treat the cells (Dejean et al., 2006). Our study found that the expression of Cyto-c is ly enhanced in cells transfected with pcDNA3.1-BMP9. During mitochondrial channel-associated apoptosis, Cyto-c can bind to the apoptosome and cleave pro-Caspase-9. Caspase-9 can then suppress tumor development by inducing cell apoptosis and death ( $\mathrm{Li}$ et al., 1997; Marsden et al., 2002; Janssen et al., 2007; Olsson and Zhivotovsky, 2011). Our in vitro analysis found that the enhanced expression of BMP9 is associated with enhanced expression of Bcl-2, Cyto-c, Caspase-9, and Caspase-3 (Figure 3).

Following the in vitro experiments, we established an OS tumor model in mice and studied the in vivo anti-tumor effect of BMP9. We used attenuated Salmonella, which has been used previously for the delivery of other therapeutic agents such as antigens, for gene delivery of pcDNA3.1 or pcDNA3.1-BMP9 (Dignam, 1990; Konjufca et al., 2006; Curtiss et al., 2010). In fact, attenuated Salmonella has been investigated for use in clinical trials for gene therapy (Lerman and Minna, 2000). Our study showed that tumors grow slower in the mice treated with pcDNA3.1-BMP9 compared to mice treated with pcDNA3.1 (Figure 4). On day 28, the mice treated with pcDNA3.1-BMP9 had a lower average tumor weight compared to mice treated with pcDNA3.1 (0.53 g compared to $0.98 \mathrm{~g}$, respectively). Mice treated with pcDNA3.1-BMP9 also had higher expression of BMP9 in the tumors compared to mice treated with pcDNA3.1. Similar to the in vitro studies, there was enhanced expression of Cyto-c, Caspase- 9 and Caspase-3 in tumor cells from mice treated with Salmonella carrying pcDNA3.1-BMP9 compared to pcDNA3.1 (Figure 4). Different from our in vitro study, we do not observe a difference in the expression of Bcl-2 between the two groups of mice. This might be attributed to the differences in the in vitro and in vivo environments. However, we did not assess in vivo toxicity and this will be examined in following research in order to identify an optimal dose of BMP9. In addition, our following studies will also assess other potential cellular pathways that might be associated in the anti-tumor process of BMP9 (Ye et al., 2008; Herrera et al., 2009; Wang et al., 2011).

Genetics and Molecular Research 15 (3): gmr.15038036 
This study examined the association between BMP9 and OS tumor development. We found increased expression of BMP9 in OS tumor tissue and MG63 cells. Transfection of cells with BMP9 resulted in decreased proliferation and increased apoptosis of OS cells. This was accompanied with increased expression of apoptotic genes (Bcl-2, Cyto-C, Caspase-3, and Caspase-9). To validate this phenomenon in vivo, tumorigenic mice were treated with BMP9, resulting in decreased tumor growth rates and size and enhanced expression of apoptosisrelated oncogenes.

\section{Conflicts of interest}

The authors declare no conflict of interest.

\section{REFERENCES}

Bielack S, Carrle D, Casali PG and Grp EGW; ESMO Guidelines Working Group (2009). Osteosarcoma: ESMO clinical recommendations for diagnosis, treatment and follow-up. Ann. Oncol. 20 (Suppl 4): 137-139. http://dx.doi. org/10.1093/annonc/mdp154

Carreira AC, Lojudice FH, Halcsik E, Navarro RD, et al. (2014). Bone morphogenetic proteins: facts, challenges, and future perspectives. J. Dent. Res. 93: 335-345. http://dx.doi.org/10.1177/0022034513518561

Chen C, Grzegorzewski KJ, Barash S, Zhao Q, et al. (2003). An integrated functional genomics screening program reveals a role for BMP-9 in glucose homeostasis. Nat. Biotechnol. 21: 294-301. http://dx.doi.org/10.1038/nbt795

Curtiss R, 3rd, Xin W, Li Y, Kong W, et al. (2010). New technologies in using recombinant attenuated Salmonella vaccine vectors. Crit. Rev. Immunol. 30: 255-270.http://dx.doi.org/10.1615/CritRevImmunol.v30.i3.30

Dejean LM, Martinez-Caballero S and Kinnally KW (2006). Is MAC the knife that cuts cytochrome c from mitochondria during apoptosis? Cell Death Differ. 13: 1387-1395.http://dx.doi.org/10.1038/sj.cdd.4401949

Dignam JD (1990). Preparation of extracts from higher eukaryotes. Methods Enzymol. 182: 194-203. http://dx.doi. org/10.1016/0076-6879(90)82017-V

Gill J, Ahluwalia MK, Geller D and Gorlick R (2013). New targets and approaches in osteosarcoma. Pharmacol. Ther. 137: 89-99.http://dx.doi.org/10.1016/j.pharmthera.2012.09.003

Herrera B, van Dinther M, Ten Dijke P and Inman GJ (2009). Autocrine bone morphogenetic protein-9 signals through activin receptor-like kinase-2/Smad1/Smad4 to promote ovarian cancer cell proliferation. Cancer Res. 69: 92549262.http://dx.doi.org/10.1158/0008-5472.CAN-09-2912

Higuchi C and Yoshikawa H (2004). [Bone morphogenetic proteins]. Nippon Rinsho 62 (Suppl 2): 52-56.

Janssen K, Pohlmann S, Jänicke RU, Schulze-Osthoff K, et al. (2007). Apaf-1 and caspase-9 deficiency prevents apoptosis in a Bax-controlled pathway and promotes clonogenic survival during paclitaxel treatment. Blood 110: 3662-3672. http://dx.doi.org/10.1182/blood-2007-02-073213

Kim M and Choe S (2011). BMPs and their clinical potentials. BMB Rep. 44: 619-634. http://dx.doi.org/10.5483/ BMBRep.2011.44.10.619

Konjufca V, Wanda SY, Jenkins MC and Curtiss R, 3rd (2006). A recombinant attenuated Salmonella enterica serovar Typhimurium vaccine encoding Eimeria acervulina antigen offers protection against E. acervulina challenge. Infect. Iтmип. 74: 6785-6796. http://dx.doi.org/10.1128/IAI.00851-06

Lerman MI and Minna JD (2000). The 630-kb lung cancer homozygous deletion region on human chromosome 3p21.3: identification and evaluation of the resident candidate tumor suppressor genes. The International Lung Cancer Chromosome 3p21.3 Tumor Suppressor Gene Consortium. Cancer Res. 60: 6116-6133.

Li P, Nijhawan D, Budihardjo I, Srinivasula SM, et al. (1997). Cytochrome c and dATP-dependent formation of Apaf1/caspase-9 complex initiates an apoptotic protease cascade. Cell 91: 479-489. http://dx.doi.org/10.1016/S0092$\underline{8674(00) 80434-1}$

Liebermann DA, Hoffman B and Steinman RA (1995). Molecular controls of growth arrest and apoptosis: p53-dependent and independent pathways. Oncogene 11: 199-210.

Liu X, Kim CN, Yang J, Jemmerson R, et al. (1996). Induction of apoptotic program in cell-free extracts: requirement for dATP and cytochrome c. Cell 86: 147-157.http://dx.doi.org/10.1016/S0092-8674(00)80085-9

Lv Z, Yang D, Li J, Hu M, et al. (2013). Bone morphogenetic protein 9 overexpression reduces osteosarcoma cell migration and invasion. Mol. Cells 36: 119-126. http://dx.doi.org/10.1007/s10059-013-0043-8

Genetics and Molecular Research 15 (3): gmr.15038036 
Marsden VS, O'Connor L, O'Reilly LA, Silke J, et al. (2002). Apoptosis initiated by Bcl-2-regulated caspase activation independently of the cytochrome c/Apaf-1/caspase-9 apoptosome. Nature 419: 634-637. http://dx.doi.org/10.1038/ nature 01101

Mei S, Theys J, Landuyt W, Anne J, et al. (2002). Optimization of tumor-targeted gene delivery by engineered attenuated Salmonella typhimurium. Anticancer Res. 22 (6A): 3261-3266.

Olsson M and Zhivotovsky B (2011). Caspases and cancer. Cell Death Differ. 18: 1441-1449. http://dx.doi.org/10.1038/ cdd.2011.30

Pang H, Flinn R, Patsialou A, Wyckoff J, et al. (2009). Differential enhancement of breast cancer cell motility and metastasis by helical and kinase domain mutations of class IA phosphoinositide 3-kinase. Cancer Res. 69: 88688876. http://dx.doi.org/10.1158/0008-5472.CAN-09-1968

Saeter G, Kloke O and Jelic S; ESMO Guidelines Task Force (2005). ESMO Minimum Clinical Recommendations for diagnosis, treatment and follow-up of osteosarcoma. Ann. Oncol. 16 (Suppl 1): i71-i72.http://dx.doi.org/10.1093/ annonc/mdi822

Song JJ, Celeste AJ, Kong FM, Jirtle RL, et al. (1995). Bone morphogenetic protein-9 binds to liver cells and stimulates proliferation. Endocrinology 136: 4293-4297.

Wang K, Feng H, Ren W, Sun X, et al. (2011). BMP9 inhibits the proliferation and invasiveness of breast cancer cells MDA-MB-231. J. Cancer Res. Clin. Oncol. 137: 1687-1696. http://dx.doi.org/10.1007/s00432-011-1047-4

Williams GT and Smith CA (1993). Molecular regulation of apoptosis: genetic controls on cell death. Cell 74: 777-779. http://dx.doi.org/10.1016/0092-8674(93)90457-2

Ye L, Kynaston H and Jiang WG (2008). Bone morphogenetic protein-9 induces apoptosis in prostate cancer cells, the role of prostate apoptosis response-4. Mol. Cancer Res. 6: 1594-1606. http://dx.doi.org/10.1158/1541-7786.MCR$\underline{08-0171}$

Genetics and Molecular Research 15 (3): gmr.15038036 